\title{
Uruguay: \\ cien años de transición demográfica
}

\author{
Adela Pellegrino
}

Cien AÑos de TRANSición

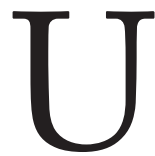

Truguay registra una historia poblacional caracterizada por la pequeñez y la precocidad en la transición demográfica. En los últimos años del siglo XIX, se observó un descenso de los niveles de mortalidad, y en los primeros años del xx, el descenso de la fecundidad, constituyendo éste un fenómeno nuevo para América Latina (Chackiel, 2004; CosioZavala, 1996).

La fecundidad fue bajando lentamente a lo largo del siglo xx; el proceso se aceleró en las últimas décadas del siglo, al punto que en 2004 se alcanzó el nivel de reemplazo (Varela, 2004).

La inmigración internacional originada en países europeos, así como 
la inmigración limítrofe, constituyeron un componente importante en el crecimiento de la población uruguaya hasta la mitad del siglo xx. Este fenómeno se revierte alrededor de la década de 1960, cuando se detiene la inmigración europea, pues una parte de los migrantes retornan a sus países y comienza la emigración de la población uruguaya, que se convirtió en un fenómeno permanente a lo largo de las cuatro últimas décadas del siglo. En dos circunstancias la emigración alcanzó un auge tan alto que el crecimiento de la población mostró valores negativos en algunos años (Pellegrino, 2011).

El descenso de la fecundidad y la mortalidad perfilaron la estructura de la población a lo largo de ese prolongado proceso de transición; los cambios fueron lentos y la sociedad se acostumbró a la nueva realidad. La migración internacional aceleró estos procesos, dado su carácter selectivo en lo referido a las edades, aumentando los tramos de jóvenes y reduciéndolos en el caso de la emigración. Se puede concluir que la trayectoria demográfica puede ser considerada diferente a la de otros países latinoamericanos, no sólo por su inicio precoz sino también por el carácter gradual de su evolución.

Desde mediados de la década de 1980 aparecen indicios de transformaciones en las características de la nupcialidad y la estructura de las familias: los matrimonios se redujeron a la mitad entre 1990 y 2007; en este mismo periodo las uniones consensuales crecieron rápidamente en todos los sectores sociales, aumentó la edad del matrimonio y el divorcio experimentó un crecimiento de gran magnitud (Cabella, 2007). Estos indicadores, unidos al descenso de la fecundidad en estos años, permiten considerar que la segunda transición demográfica (Van de Kaa, 1997 y García y Rojas, 2003) se ha consolidado en el país.

Uruguay no tuvo que enfrentar los desafíos del crecimiento explosivo de la población como ocurrió en la mayoría de los países latinoamericanos. A pesar del carácter gradual en su evolución, el efecto del envejecimiento de la estructura de edades impulsó una revisión de las políticas sociales, de las políticas de salud y de seguridad social, para ha- 
blar de algunos de los asuntos más importantes derivados de este tema. Una sociedad con una estructura de edades envejecida necesita generar transformaciones profundas que van desde las características de la familia hasta el hábitat y, sobre todo, suponen cambios importantes en las relaciones entre las generaciones.

¿A qué causas responde la precocidad en los cambios demográficos en Uruguay con respecto a América Latina y otras regiones del mundo? Uruguay se anticipó a los fenómenos que caracterizan la transición demográfica al menos en treinta años respecto del resto de los países latinoamericanos, algunos de los cuales comenzaron su transición demográfica hacia 1930, pero la mayoría en las décadas de 1950 y 1960. Sólo Argentina tuvo una cronología similar a la uruguaya, pero restringida a la Provincia de Buenos Aires.

La literatura académica debate aún sobre las causas de la transición demográfica y, en particular, sobre el descenso de la fecundidad en los países del noroeste de Europa, donde se inició. ¿La causa de los cambios está en los procesos econó- micos y sociales que revolucionaron Europa desde fines del siglo xvin? ¿Los procesos son consecuencia de estos cambios? ¿O se trata de un fenómeno autónomo? (Lee y Reher, 2011). Fue en las últimas décadas del siglo XIX cuando en Uruguay empezó a evidenciarse el descenso de la mortalidad, fenómeno que en la mayoría de las regiones se consideró el inicio de la transición demográfica. De manera similar a Argentina, Uruguay se incorporó al circuito de la fase de la globalización, que tuvo su auge en las últimas décadas del siglo xix y primeras décadas del siglo xx. Esta integración le permitió participar en las rutas del comercio internacional, en las inversiones internacionales y, con ellas, se sumó la inmigración de ultramar. Estos factores fueron asociados a otros fenómenos sociales y culturales que incorporaron a la sociedad uruguaya mentalidades y comportamientos «modernos», reflejados a su vez en el cambio de la familia, la relación entre los sexos y las actitudes frente a la reproducción.

¿Fue la inmigración la que impulsó los cambios en el comporta- 
miento y en la reproducción? ¿Fueron los inmigrantes el vehículo de difusión de la innovación? Parece evidente que para que los cambios innovadores arraiguen debe existir el caldo de cultivo necesario en la sociedad.

La trayectoria europea fue considerada modélica y las diferencias con respecto a otros procesos fueron conocidas como anomias. Sin embargo, las evidencias surgidas del avance de la investigación concluyeron que las cronologías, las trayectorias y, sobre todo, sus causas y consecuencias fueron diferentes entre regiones. La transición «precoz», tal cual se realizó en países del sur de América, tuvo trayectorias diferentes; muchas deben ser aún investigadas.

Uruguay se caracterizó por el «vacío poblacional», pues tuvo una impronta urbana desde sus orígenes; la integración de sus pobladores fue una mezcla de grupos diferentes: los originarios del territorio, los indígenas trasladados al territorio por la disolución de las misiones jesuíticas, los africanos traídos como esclavos, los colonizadores españoles y portugueses $y$, luego, la inmigración europea, en este caso principalmente del sur de Italia, España y el sur de Francia.

La sociedad que se consolidó fue heterogénea, con pocas tradiciones a las cuales enfrentarse. La inmigración europea masiva de fines del siglo xIx y principios del siglo xx provenía del sur de Italia y de regiones de España que todavía registraban altas tasas de fecundidad en el periodo en que se produjeron los traslados. Pero los inmigrantes constituían una masa heterogénea en la que se mezclaban campesinos en busca de tierras con aquéllos que abandonaban Europa para escapar del medio rural y encontrar la ciudad en América, obreros con experiencia sindical y militantes políticos socialistas o anarquistas, cuyo pensamiento se difundió en los sindicatos, en la prensa militante y de las organizaciones de inmigrantes, en la cual los temas relacionados con el control de la fecundidad no estaban ausentes (Pellegrino, 1997).

Por otra parte, desde los últimos años del siglo xIx y los primeros del siglo $\mathrm{xx}$ se implementaron medidas que alentaron condiciones que per- 
mitieron el cambio demográfico: las medidas relativas a la salud pública y a la sanidad condujeron a una baja mortalidad temprana y la reforma educativa impulsó un acceso más igualitario a la educación, no solamente entre los sectores sociales, sino también entre los sexos. Muy tempranamente, los datos informan sobre la tendencia a la equiparación entre los niveles de alfabetización de hombres y mujeres. Estos temas, entre otros, condujeron también a una demografía que avanzaba hacia los cambios demográficos.

En las primeras décadas del siglo xx el gobierno de José Batlle tuvo una importancia fundamental en el perfil que asumió Uruguay como país democrático e igualitario. Se tomaron medidas fundamentales en lo relativo a la igualdad entre los hombres y las mujeres, medidas de salud que impulsaron el combate a la mortalidad infantil y medidas relativas a la infancia. La mortalidad infantil en Uruguay a principios del siglo xx mostraba niveles similares a los de Francia.

En la segunda década del siglo xx la fecundidad comenzó a descen- der. La tasa global de fecundidad, estimada por Pollero (1994), era de seis en 1908, se ubicó en tres desde 1930 hasta 1970. También la mortalidad se estabilizó en ese periodo. La investigación demuestra que el importante impulso inicial de la lucha contra la mortalidad infantil no avanzó, y que Uruguay, antes pionero en el descenso de la mortalidad infantil en América del Sur, fue siendo alcanzado por otros países.

La segunda mitad del siglo $\mathrm{xx}$ fue también el escenario de cambios demográficos importantes. El crecimiento de la población continuó reduciéndose debido al descenso de la tasa de la natalidad y de la tasa de migración internacional. Ésta última adoptó un signo negativo de manera persistente a lo largo del periodo y en algunos años la emigración fue tan alta que el crecimiento vegetativo no logró compensar la pérdida de población, provocando su decrecimiento (Pellegrino, 2003).

La tasa bruta de mortalidad crece muy moderadamente desde mediados del siglo hasta fines de la década de 1970, y luego permanece 
estancada. El gráfico 1 pone de manifiesto la reducción de la tasa de crecimiento natural de la población durante la segunda parte del siglo $x x, y$ a la vez muestra el importante efecto de los movimientos migratorios para explicar el crecimiento total de la población.

\section{GRÁFICA I}

Tasas brutas de natalidad y mortalidad, tasa neta de migración y tasa de crecimiento total (Uruguay, 1952-2005).

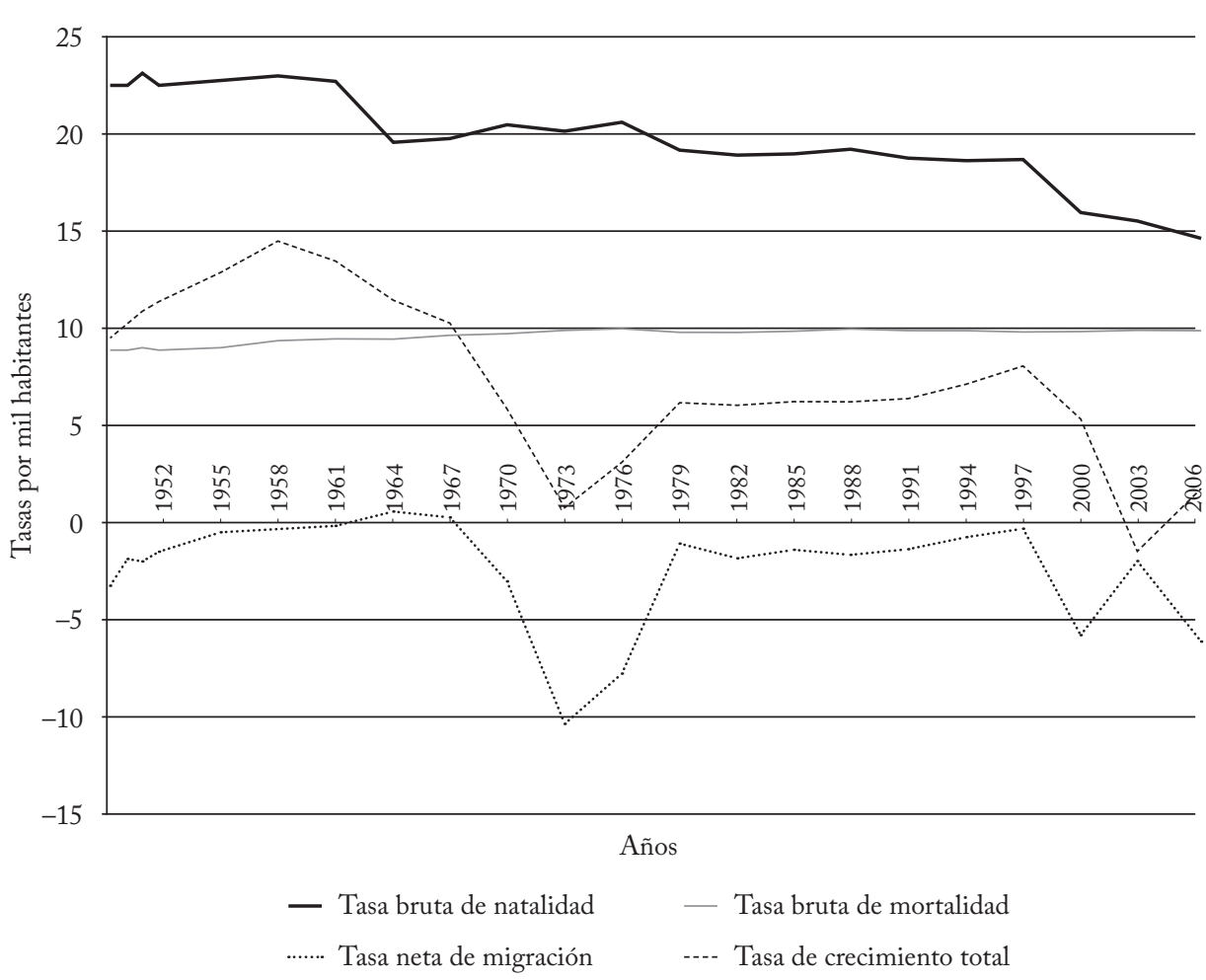

Fuente: Programa de población. 
Entre las consecuencias más relevantes de la transición demográfica, se encuentran los cambios producidos en la estructura de edades; su importancia ha sido tal que algunos demógrafos los han reunido bajo el término «transición estructural de las edades» (Pool et al., 2005). Este concepto busca poner énfasis en los efectos del proceso de reducción progresiva de las cohortes de nacimiento a partir de los cambios generados por el descenso de la fecundidad, por la mejora de la mortalidad - en menor medida-y, en algunos países, por los efectos de la migración. La transición estructural de las edades se concentra en los efectos de la transición demográfica sobre la estructura de edades, destacando el pasaje de poblaciones relativamente jóvenes hacia otras caracterizadas por el envejecimiento demográfico (Cabella y Pellegrino, 2009).

A mediados del siglo xx, algunos estudiosos de las ciencias sociales e informes que se hicieron sobre Uruguay demostraron que aparecían los primeros signos de envejecimiento, que fueran identificados inicialmente por Solari (1957) y luego en el Informe de la Comisión de Inversiones y Desarrollo Económico (CIDE) (Cataldi, 1964).

El censo de 1908 demostró que $2.9 \%$ de las personas de 65 años y más eran el porcentaje del total. E1 censo de 1963 mostró que ese porcentaje era $7.6 \%$ y actualmente el último censo de 2011 mostró que el porcentaje es de $14.1 \%$, evidenciando que el proceso de envejecimiento había avanzado durante el siglo (gráfico 2).

Durante la mayor parte de la última mitad del siglo la reducción de la fecundidad fue la variable que más contribuyó al envejecimiento de la población, en la medida en que determinó la reducción progresiva del peso demográfico de los niños y los jóvenes. Este proceso se conoce con el nombre de envejecimiento «por la base» de la pirámide. Actualmente, a este proceso se suma el envejecimiento "por la cúspide», ello significa que a medida que los progresos en la mortalidad se concentran en la población de mayor edad, ésta sobrevive por más tiempo y por lo tanto representa 
una porción mayor de la población total; incluso al interior del grupo de adultos mayores las franjas etarias más altas cobran mayor importancia. ${ }^{1}$ La migración internacional ha aportado al decrecimiento de la población durante la última mitad del siglo pasado en la medida que el saldo ha sido negativo durante ese periodo.

\section{GRÁFICA 2}

Evolución de la estructura de edades de Uruguay.

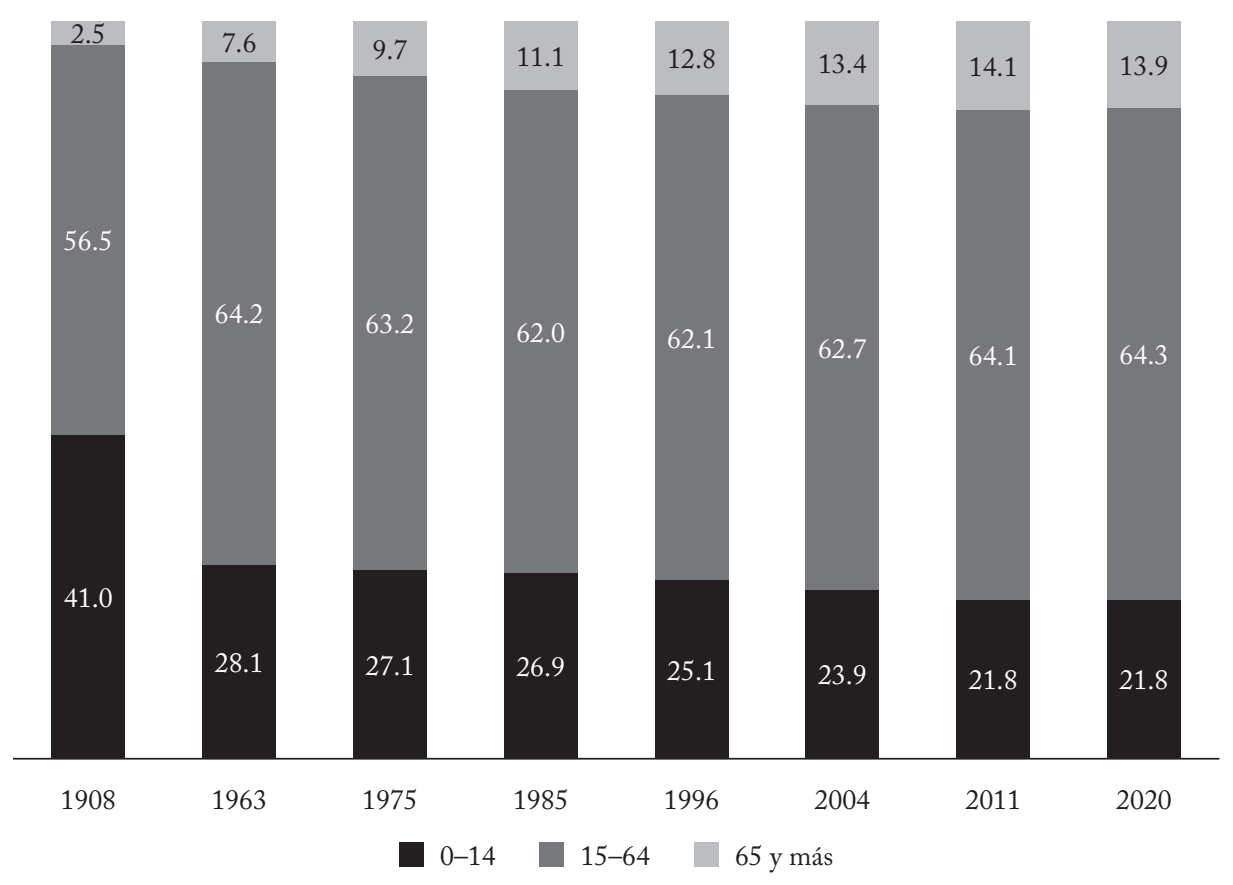

Fuente: Censos nacionales de población 1908-2011 y Proyecciones de población (INE).

${ }^{11 /}$ El envejecimiento por la cúspide debido al aumento de cohortes de edades numerosas que nacieron en el periodo del alto crecimiento fue otra causa importante de incremento de las edades a partir de 65 y más. Ese fenómeno fue menos perceptible en el caso de Uruguay dado el carácter gradual de la transición de la fecundidad en este país. 
La lentitud con que se procesó la reducción en la tasa de crecimiento de la población uruguaya propició un equilibrio favorable entre las edades, permitiendo el sostenimiento de relaciones de dependencia positivas en términos relativos a los observados en esos años entre los otros países latinoamericanos. La gradualidad del descenso de la fecundidad explica el bajo potencial de crecimiento del país y por consiguiente, aunque no cuenta con el llamado bono demográfico, ha mantenido una relación de dependencia favorable.

De acuerdo a las proyecciones nacionales de Uruguay que realizó el Instituto Nacional de Estadísticas (INE) se espera un lento crecimiento de la población, aumento del envejecimiento de la estructura de edades, reducción de la tasa de natalidad y de la fecundidad, descenso de la mortalidad infantil y aumento de la esperanza de vida.

\section{Oportunidades y DESAFíos}

DE UNA SOCIEDAD ENVEJECIDA

El proceso de envejecimiento de la población es una de las conquistas sociales más importantes del siglo xx. El progreso en el cuidado de la salud que ha contribuido a alargar la vida y a aumentar la esperanza de vida puede considerarse como un triunfo importante de la humanidad. Sin embargo, el envejecimiento de la población también ha sido objeto de preocupaciones por los gobiernos y observadores de la sociedad. Las consecuencias del crecimiento de las edades avanzadas implican el aumento del costo de jubilaciones y pensiones, así como del gasto en salud. Ello se suma, debido a los aumentos de la esperanza de vida de los adultos mayores, a que se incrementen los requerimientos de atención, diagnósticos y tratamientos más caros de sobrevivientes a edades con mayores discapacidades (Ham Chande, 2001). Estos temas, entre otros, han sido considerados como uno de los problemas importantes por resolver en la actualidad y en el futuro.

El envejecimiento es uno de los efectos de la transición demográfica avanzada. Todos los componentes que integran la dinámica demográfica pueden incidir para que la 
estructura de edades incremente sus tramos más altos de la pirámide.

En el mediano y largo plazo el envejecimiento de las poblaciones es inevitable en los países que han avanzado en la transición demográfica. Las políticas posibles para revertir esta tendencia o sus efectos son de dos tipos. En primer lugar, las políticas que se proponen modificar los factores estructurales que componen la dinámica demográfica, como el objetivo de estimular la fecundidad o la migración internacional.

En segundo lugar, se proponen medidas que permitan adaptar los servicios así como medidas de intervención que debe realizar el Estado frente a una población envejecida cuyo peso es creciente y puede ser más importante en el futuro. Los temas que más se han debatido son los relativos a las transferencias entre las generaciones, incluyendo el financiamiento de las pensiones y jubilaciones y los cambios necesarios en el sistema de salud.

¿Cuáles serían las medidas posibles para enfrentar el envejecimiento de la estructura de edades en el caso de Uruguay?
Durante muchas décadas Uruguay fue considerado por varios de los gobiernos nacionales $y$, fundamentalmente, por los organismos internacionales como un país sin problemas poblacionales, dado que el tema central era luchar contra el crecimiento de la población y las políticas se orientaban al control de la fecundidad. Fue considerado un ejemplo entre los países latinoamericanos por sus indicadores demográficos y, sobre todo, por su larga historia de descenso de la fecundidad. Actualmente, la situación ha cambiado. La estructura de la población uruguaya puede ser considerada, más bien, como la antesala de lo que habrán de atravesar algunos países de la región, ya que se encuentra en las últimas fases de la transición demográfica o ya ha entrado en la llamada segunda transición, con los problemas relacionados con la estructura de edades y el envejecimiento.

Políticos y estudiosos de la sociedad se preguntan si es importante para el futuro del país estimular el crecimiento de la población, en particular de los jóvenes. En ese 
caso, ¿qué medidas son las adecuadas y posibles?

Medidas de fondo posibles son las destinadas a cambiar la tendencia descendente de la fecundidad y la migración internacional. Estas medidas podrían incidir en temas muy sensibles para la sociedad. Las políticas natalistas han sido adoptadas por regímenes de signo diverso, desde algunos países de la Europa del Este hasta la Alemania nazi, pasando por la Francia de la segunda postguerra. En general, se puede afirmar que dichas políticas han estado asociadas a proyectos nacionalistas y de afirmación de la nación (Pellegrino y Calvo, 2008).

Actualmente, muchos países desarrollados han implementado políticas de estímulo económico a los nacimientos y ventajas fiscales a las familias numerosas, como también reducción del número de años necesarios para alcanzar la jubilación, en general para las madres. Otras políticas fueron destinadas a facilitar la maternidad desde diversos aspectos, como, por ejemplo, las políticas para integrar el trabajo fuera del hogar con la fecundidad
— guarderías, licencias maternales o paternales por periodos largosque apoyan a las parejas - especialmente a las mujeres trabajadorasque desean tener hijos.

En la discusión sobre las políticas natalistas están presentes tanto los aspectos relativos a los valores y las consecuencias sociales de dichas políticas como la evaluación de la eficacia de las medidas propuestas o implementadas. La envergadura de la cuestión implica que toda evaluación de los temas esté impregnada de concepciones y puntos de vista políticos, filosóficos y éticos (Pellegrino y Calvo, 2008).

Las políticas planteadas en los países desarrollados, con respecto al crecimiento de la población y a evitar el detener el envejecimiento, han tenido consecuencias diferentes. En el caso de Suecia y Francia las medidas políticas permitieron que la tasa de fecundidad se ubicara entre las mayores de Europa. Estas medidas son acciones que han tenido permanencia en el tiempo y sus resultados han sido observados en el largo plazo.

En Suecia, las políticas no tuvieron un objetivo explícito de estímu- 
lo al aumento de la fecundidad, las políticas impulsadas fueron realizadas en el marco de las políticas de apoyo a la familia y a las mujeres en su objetivo de alcanzar la equidad de género y la combinación posible entre la crianza de los hijos con el trabajo remunerado de los dos miembros de la pareja (Pardo, Varela y Pollero, 2011).

En Francia, que es un país con larga tradición de objetivos natalistas, como se ha mencionado, la tasa de fecundidad se ubica entre las más altas de Europa. Los objetivos natalistas se han integrado a una larga tradición de políticas familiares que se han orientado también a la corresponsabilidad de los hombres y las mujeres en las familias.

Otros países europeos como Italia y España que han aprobado medidas de estímulo al aumento de la fecundidad, las han implementado en un periodo más reciente y aún no se pueden ver sus resultados.

En el caso de Uruguay, el establecimiento de las asignaciones familiares (AFAM) en la década de 1940 tuvo en su fundamento un objetivo natalista, aunque no esta- mos en condiciones de evaluar en qué medida esto influyó en el nivel de la fecundidad, que se mantuvo en alrededor de tres hijos por mujer hasta la década de 1970. La devaluación de las asignaciones familiares hizo que su peso sobre los ingresos de los hogares dejara de tener un efecto sobre el número de hijos. Ese tema ha cambiado recientemente a partir de la inclusión y la modificación de las asignaciones familiares dentro del Plan de Equidad del Ministerio de Desarrollo Social y aún no tenemos evaluaciones de sus consecuencias.

Las encuestas que se han realizado en Uruguay con el objetivo de indagar sobre el número de hijos ideal en las mujeres o en las familias permiten concluir que, en los sectores altos y medios, las mujeres declaran que desean tener un número de hijos mayor que el que realmente tienen, mientras que las mujeres con ingresos bajos o con menos educación desean menos hijos de los que tienen (Ministerio de Salud Pública-Organización Panamericana de la Salud, 1994; Pardo y Peri, 2006). Estas respuestas permiten 
sostener que hay una insatisfacción en la población uruguaya en lo relativo a la reproducción y que las políticas orientadas a los sectores con altas carencias deben enfatizarse a través de los programas de educación sexual y de salud reproductiva.

En el caso de los sectores medios se debería avanzar en el conocimiento de sus expectativas en esta materia para comprender mejor sus decisiones reproductivas. En todo caso, las intervenciones que permitan integrar el trabajo remunerado con la fecundidad aparecen como necesarias para que tener hijos sea un acto realizado en mejores condiciones, independientemente de que ello se traduzca o no en un incremento de la natalidad (Pellegrino y Calvo, 2008).

En los últimos años estos temas han entrado, de manera tímida, en el ámbito político, y se han tomado algunas medidas orientadas a encontrar el equilibrio entre la reproducción y el trabajo, se ha ampliado la duración de las licencias por nacimiento tanto a las madres como a los padres, y se debate acerca de la instalación de un sistema nacional de cuidados.
La migración internacional es otro componente que puede actuar en el crecimiento o decrecimiento de la población, así como en la distribución por edades. Algunos países de transición avanzada han buscado inmigrantes buscando equilibrar la población activa y la pasiva, así como satisfacer la demanda de trabajadores en déficit.

Las políticas de estímulo a la inmigración son de tipo diferente. Los países tradicionales, receptores de inmigrantes como Canadá, Estados Unidos y Australia, han impulsado cuotas por nacionalidades de origen, de trabajadores y especialistas pero también buscando incorporar población para integrar a sus naciones. En el caso de muchos de los países europeos, se buscó tratar de integrar especialistas o científicos y trabajadores contratados por periodos precisos, para suplir los déficits de trabajadores sin objetivos poblacionistas. Actualmente, el desempleo, consecuencia de la crisis económica, y el malestar entre los inmigrantes y los nativos han terminado en políticas migratorias de cierre a las fronteras. 
En el caso de Uruguay, la migración ha tenido un impacto fundamental, tanto desde el punto de visto demográfico como desde el punto de visto económico y cultural. Durante la primera fase de globalización económica, Uruguay fue destino de una parte de los europeos que se desplazaron para instalarse en el continente americano y otras regiones de África y Oceanía. La inmigración del siglo xix y de la primera mitad del siglo $\mathrm{xx}$ fue un componente importante del crecimiento demográfico y tuvo efectos relevantes en el mercado de trabajo, la educación, el arte y la cultura del país.

En la segunda globalización, Uruguay también participa a la intensidad de la movilidad de población, ahora en condición de país de emigración hacia los países de América del Norte y Europa. Durante ese periodo tuvo consecuencias importantes en el decrecimiento de la población.

En los últimos años, debido a la crisis económica internacional que ha afectado el mercado de trabajo de los países receptores de inmigrantes, así como un periodo de algunos años de recuperación y crecimiento económico en Uruguay, se han observado flujos de migrantes de retorno, así como una presencia incipiente de inmigrantes.

La migración actual demuestra una variedad mucho mayor de modalidades migratorias, tanto en lo que se refiere a la duración de las estadías como a la frecuencia de los movimientos y a la multiplicidad de las residencias. Una modalidad que se generaliza es la de trabajar en proyectos que se desarrollan fuera de fronteras, permaneciendo en los países de origen. La utilización de medios telemáticos permite la comunicación «virtual» y genera situaciones que no son migración en sentido estricto, pero tienen implicancias importantes en la movilidad de las personas. La emigración y la inmigración pueden formar parte de un proceso general que implique flujos de signo positivo y negativo de manera simultánea. Las nuevas realidades permiten que los movimientos sean más reversibles, que se incrementen los retornos, que exista una mayor tendencia de las personas a tener varias residencias y que 
aumente el número de personas que mantienen trabajos a distancia, trabajando en un país - o en variosy residiendo en otro.

Los efectos de la migración internacional sobre el futuro de la población uruguaya son inciertos, $y$ es posible que se afirme la tendencia creciente de la inmigración al tiempo que se mantiene una emigración de jóvenes, estimulados por las redes de migrantes consolidadas en periodos anteriores.

En los años recientes la situación de la migración internacional es incierta, dada la crisis económica en los países de destino. Por otra parte, el desempleo en Uruguay ha descendido a niveles muy bajos creando una situación no propicia a la emigración e, incluso, están surgiendo nuevos flujos de inmigración.

Las políticas recientes orientadas a la migración internacional no han tenido un objetivo "poblacionista». La nueva ley 18.250 aprobada en 2008 fue redactada con el objetivo de aprobar una norma que regule el tema, basado en principios de los derechos de los migrantes y tratando de evitar algunos artículos de la ley de 1937 que implicaba la segregación de algunos grupos étnicos.

La ley está orientada a regular la situación de los inmigrantes en el país y permite crear una normativa propicia a la inmigración. ${ }^{2}$

$\mathrm{El}$ retorno de los emigrantes es un tema que ha estado presente y los datos observan un crecimiento en estos años. La ley ha incorporado un estímulo al retorno y en el Departamento del Ministerio Relaciones se ha organizado con el objetivo de regular y asesorar a los proyectos de retorno, así como asistencia a los deportados o personas que atravesaban por situaciones vulnerables.

En síntesis, la política migratoria de los dos últimos gobiernos estaba basada en el impulso a los vínculos entre los emigrantes y los proyectos con el país. Luego de haber desarrollado medidas que apoyaron el

${ }^{12 /}$ Algunos artículos de la ley están orientados a eliminar algunos obstáculos para ingresar pertenencias personales y profesionales libres de impuestos y gravámenes. 
retorno tras la caída de la dictadura militar, en este periodo se ha dado prioridad a la recuperación de las formaciones y las capacidades perdidas con la emigración, buscando la colaboración a distancia.

Las medidas necesarias para adaptar los servicios y atender a una población creciente de edades envejecidas han sido incluidas en la agenda del ámbito político, así como del académico, y se ha traslado a los medios de comunicación.

Los vínculos entre las generaciones es un tema importante a encarar en las sociedades actuales. Las relaciones entre los jóvenes y los mayores suponen un encare multidisciplinario en las políticas que se deben proyectar.

Los estudios sobre las transferencias económicas entre las generaciones son los que han avanzado en su análisis y han concluido que, tanto las privadas como las públicas, favorecen a la población mayor de 65 años más que a los menores de 18 años y menos. Furtado (2005) sostiene que el sistema de seguridad social constituye el principal mecanismo de transferencias públicas en Uruguay, y las evidencias encontradas en su estudio indican que las generaciones de trabajadores tienen una doble carga: por un lado, enfrentan su propia subsistencia y la de sus hijos y, por otro, contribuyen a la de sus padres.

Buchelli, González y Olivieri (2010) concluyen en 2006 que la asignación neta de recursos públicos a los distintos grupos de edades suponen flujos desde las edades medias a los niños y a las personas mayores; que los flujos a los mayores son superiores que los que dirigen a los niños y que en el periodo (1996-2006) se redujo la brecha de transferencias netas entre los grupos debido al aumento de la inversión pública en educación y al descenso del gasto público en pensiones, como consecuencia de la reforma de la seguridad social.

En los últimos años, el aumento del porcentaje del presupuesto a la educación, así como otros programas orientados a los niños y los jóvenes que se han desarrollado puede haber reducido la brecha entre las edades pero su evaluación no se ha realizado aún. Actualmente está 
en discusión un proyecto orientado a un sistema nacional de cuidados de las personas dependientes. E1 proyecto pretende sostener a los diferentes grupos de edades en sus necesidades recíprocas de apoyo y, de realizarlo, puede considerarse un avance fundamental de convivencia de las generaciones.

El sistema de seguridad social de Uruguay es el más antiguo de América Latina, dentro de éste el programa de las jubilaciones y pensiones tiene un lugar predominante. El Estado comenzó a aprobar las primeras pensiones en los últimos años del siglo xix y a la mitad del siglo xx el sistema se había consolidado y conseguido universalizarlo en lo relativo a la protección de la vejez. Luego de un dilatado proceso de incorporación de los distintos sectores de trabajadores, el sistema de pasividades y pensiones logró cubrir prácticamente a todos los potenciales beneficiarios (Sienra, 2007).

El crecimiento del sistema de seguridad social fue contemporáneo a cambios demográficos. Iniciado en una etapa donde la población tenía una estructura de edades en la que predominaban los jóvenes, pasada la segunda mitad del siglo $\mathrm{xx}$, Uruguay ya presentaba una estructura demográfica envejecida y se transformaba en un país de emigrantes. Entre un escenario y otro, Uruguay experimentó dos grandes procesos demográficos: la primera y la segunda transición demográfica (Pellegrino et al., 2008).

$\mathrm{El}$ proceso fue largo en relación con otros países latinoamericanos que están enfrentando el envejecimiento de la estructura de edades en un periodo breve. El gradualismo sobre el proceso podría haber permitido adaptar las reformas a los cambios demográficos; aún muchos críticos del sistema de previsión consideran que los cambios tendrían que haber advertido su adaptación al aumento del número de personas que llegan a la edad de recibir la pasividad. La reforma que fue propuesta en la década de 1990 y la ley que fue aprobada en 1995 tuvieron un espacio importante de discusión que implicó una amplia repercusión en la sociedad uruguaya.

La discusión del sistema de pensiones y jubilaciones giró en torno a 
si el sistema debería ser de reparto o de capitalización; también se discute aún el tema de la edad que debe alcanzar un individuo para pasar de activo a pasivo.

Uno de los principales problemas que enfrenta el país en materia de seguridad social es que una parte significativa de la población encontrará inconvenientes importantes para cumplir con los requisitos jubilatorios debido a las dificultades que supone alcanzar el tiempo de cotización requerido por el sistema de previsión. De acuerdo con las proyecciones presentadas por $\mathrm{Bu}-$ cheli et al. (2006), la intermitencia en los periodos de cotización en el mercado de empleo formal (por desempleo o informalidad) constituyen barreras importantes para lograr los beneficios jubilatorios.

\section{REFLEXIONES FINALES}

El envejecimiento de la estructura de edades de la población debe ser considerado como un logro de la sociedad, no como un fracaso. Este fenómeno es creciente en Uruguay: las proyecciones de población, aún en las versiones más moderadas, prevén un aumento importante del peso de los mayores de 65 años en el futuro.

La «cuestión demográfica» ha ocupado progresivamente espacios significativos en la discusión sobre el futuro del país y es necesario estimular la sensibilización frente al tema por parte de quienes se encargan del presente y piensan el futuro. Si bien el Uruguay ha sido considerado como un país sin problemas, su historia de la segunda mitad del siglo xx demuestra que la «explosión demográfica» y el alto crecimiento de la población no son los factores más importantes del freno al desarrollo. Los países con poco crecimiento de la población han tenido trayectorias similares, peores o mejores, que las de los países de alto crecimiento; sin embargo, son los proyectos y las opciones realizadas por las naciones las que prefiguran sociedades igualitarias y prósperas. Los países pequeños en el número de la población, como Uruguay, deben construir sus proyectos ubicados en sus dimensiones $y$ en su contexto a fin de encontrar 
un camino posible y positivo para sus habitantes.

Proponer leyes y reglas de funcionamiento, así como crear un ambiente espiritual propicio para la

\section{REFERENCIAS}

Amarante, Verónica y Alma Espino (2007), «Informalidad y protección social en Uruguay. Elementos para una discusión conceptual y metodológica», Documentos de Trabajo, Montevideo, Instituto de Economía, número 1/07.

Azar, Paola, Sandra Rodríguez y Claudia Sanguinetti (2001), «Análisis sobre la duración del Desempleo (Uruguay 19861999)», Documentos de Trabajo, Montevideo, Instituto de Economía, número 9/01.

Birn, Anne-Emanuelle, Wanda Cabella y Raquel Pollero (2003), «La mortalidad infantil uruguaya en la primera mitad del siglo xx: un análisis por causas del convivencia entre las diferentes generaciones es uno de los desafíos de una sociedad envejecida y debe, por tanto, ser un objetivo importante en el diseño de políticas públicas.

pasado al presente», Anales de las VII Jornadas Argentinas de Estudios de Población, Tafi del Valle, Tucumán, volumen I.

Bucheli, Marisa, Natalia Ferreira-Combra, Álvaro Forteza y Ianina Rossi (2006), «El acceso a la jubilación o pensión en Uruguay ¿Cuántos y quiénes lo lograrían?», Serie Estudios y Perspectivas, Montevideo, CEPAL. Bucheli, Marisa, Cecilia González y Cecilia Olivieri (2006), «Transferencias del sector público a la infancia y la vejez en el Uruguay, 1994-2006», Notas de Población, Chile, CELADE-CEPAL, número 90.

Cabella, Wanda (2007), «El cambio familiar en Uruguay: una breve reseña de las tendencias recientes», Cuadernos del UNFPA, 
Serie Divulgación, Montevideo, Fondo de Población de Naciones Unidas, Trilce, año 1, número 1. (2009), «Dos décadas de transformaciones de la nupcialidad uruguaya: la convergencia hacia la segunda transición demográfica», Estudios demográficos y urbanos, volumen 24, número 2. y Adela Pellegrino (2009), «El envejecimiento de la población y la transición estructural de las edades» en Gabriel Bucheli y Silvana Harriet (coordinadores), $\mathrm{La}$ seguridad social en el Uruguay. Contribuciones a su bistoria, Montevideo, Universidad de la República.

CAtaldi, Alberto (1964), La situación demográfica del Uruguay en 1957 y proyecciones a 1982, Chile, CELADE.

Chackiel, Juan (2000), «El envejecimiento de la población latinoamericana, ¿hacia una relación de dependencia favorable?», Serie Población y Desarrollo, Chile, CEPAL-CELADE, número 4. , «La transición de la fecundidad en América Latina 1950-2000», Papeles de Población, número 41.
—_ «La dinámica demográfica en América Latina», Serie Población $y$ Desarrollo, Chile, cepal-celaDE, número 52.

Damonte, Ana María (1994), «La transición de la mortalidad en Uruguay», Documentos de trabajo, Unidad Multidisciplinaria, Facultad de Ciencias Sociales, Montevideo, número 17.

Ham Chande, Roberto (2001), «Esperanza de vida y expectativas de salud en las edades avanzadas», Estudios demográficos $y$ urbanos, número 048.

Hatton, Timothy J. y Jeffrey G. Williamson (1998), The Age of Mass Migration, Nueva York, Oxford University Press. Informe cIDE (1964), Montevideo. Lee, Ronald D. y David S. Reher (2011), «Demographic Transition and Its Consequences», Population and Development Review, volumen 37.

Lesthaeghe, Ron (1995), «The Second Demographic Transition in Western Countries: An interpretation» en Karen Oppenheim Mason y An-Magritt Jensen (editores), Gender and Family 
Change in Industrialized Countries, Oxford, Clarendon Press.

Furtado, Magdalena (2005), «Las transferencias intergeneracionales en Uruguay», Notas de Población, Chile, CELADE-CEPAL, número 80.

Ministerio de Salud Pública-Organización Panamericana de la Salud (1994), Mujer y Fecundidad en Uruguay, Factores determinantes directos de la fecundidad y sus implicancias en Salud, Montevideo, Trilce.

Pellegrino, Adela, Wanda Cabella, Mariana Paredes, Raquel Pollero y Carmen Varela (2008), «De una transición a otra: la dinámica demográfica del Uruguay en el siglo xx» en $\mathrm{B}$. Nahum, Uruguay en el siglo XX: la sociedad, Montevideo, Ediciones de Banda Oriental. y Juan José Calvo (2007), «¿Qué hacer con la población en Uruguay? Los desafíos demográficos de las próximas décadas» en Rodrigo Arocena y Gerardo Caetano, Uruguay Agenda 2020, Montevideo, Taurus. (2003), Caracterización demográ- fica del Uruguay, Montevideo, UNFPA-Facultad de Ciencias Sociales, Universidad de la República. (1998), «Vida conyugal y fecundidad en la sociedad uruguaya del siglo Xx: una visión desde la demografía» en J. P. Barrán, G. Caetano y T. Porzecanski, Historia de la Vida Privada en el Uruguay, Montevideo, Taurus, tomo 3. Peri, Andrés (2004), «Dimensiones ideológicas del cambio familiar en Montevideo", Papeles de Población, volumen 10. e Ignacio Pardo (2006), «Nueva evidencia sobre la hipótesis de la doble insatisfacción, ¿cuánto falta para que toda la fecundidad sea deseada?», ponencia presentada en las $\mathrm{v}$ Jornadas de Investigación de la Facultad de Ciencias Sociales.

Pollero, Raquel (1994), «Transición de la Fecundidad en el Uruguay», Documentos de trabajo, Montevideo, Unidad Multidisciplinaria, Facultad de Ciencias Sociales, número 17.

Pool, Ian, Vipan Prachuabmon y Shripad Tuljapurkar (2005), «Age-structural transitions, 
populations waves and political arithmetick» en Shripad Tuljapurkar, Ian Pool y Vipan Prachuabmoh (editores), Population, Resources and Development: Riding the Age Waves, volume 1 of the new IUSsP series on population studies, Springer Verlag. Sienra, Mariana (2007), «La evolución del sistema de seguridad social en el Uruguay (1829-1986)», Documentos de trabajo, Montevideo, Instituto de Economía, número 7/07.

Solari, Aldo (1957), «El fenómeno del envejecimiento de la población uruguaya», Revista Mexicana de Sociología, Instituto de Investigaciones Sociales de la UNAM, volumen XIX, número 2. Varela Petito, Carmen (2004), «El reemplazo de la población en Uruguay, un fenómeno ausente en la agenda estatal», La fecundidad en América Latina, ¿̇transición o revolución?, Chile, CEPAL.
(2007), «Fecundidad: propuestas para la formulación de políticas. Importante pero urgente» en Juan José Calvo y Pablo Mieres (editores), Politicas de población en Uruguay, Montevideo, Fundación Rumbos, Fondo de Población de las Naciones Unidas (UNFPA).

Veronelli, Juan Carlos (2000), «Envejecimiento y condiciones educativas y laborales del adulto mayor en Uruguay», Encuentro latinoamericano y caribeño sobre las personas de edad, Chile, CEPAL, Serie Seminarios y Comentarios, número 2.

Zavala de Cosio, María Eugenia (1996), «The demographic transition in Latin America and Europe» en José Miguel Guzmán, Susheela Singh, Germán Rodríguez y Edith A. Pantelides (editores), The Fertility Transition in Latin America, Oxford, Clarendon Press. 